Case Report

\title{
"Isolated" Amelogenesis Imperfecta Associated with DLX3 Mutation: A Clinical Case
}

\author{
Anne-Laure Bonnet $\mathbb{D}^{1,2}$ Kevin Sceosole, ${ }^{3}$ Arabelle Vanderzwalm, ${ }^{3}$ Caroline Silve, ${ }^{4}$ \\ Anne-Margaux Collignon, ${ }^{1,3}$ and Celine Gaucher ${ }^{1,4,5}$ \\ ${ }^{1}$ Université de Paris, EA2496, Montrouge, Paris F-92120, France \\ ${ }^{2}$ Department of Odontology, AP-HP, Sorbonne Université, Hospital Charles Foix, Paris F-94120, France \\ ${ }^{3}$ Department of Odontology, AP-HP, Nord-Université de Paris, Hospital Louis Mourier, Paris 92700, France \\ ${ }^{4}$ Department of Genetic and Molecular Biology, AP-HP Centre-Université de Paris, Hospital Cochin, Paris F-75014, France \\ ${ }^{5}$ Department of Odontology, AP-HP, Hôpitaux Universitaire Henri Mondor, Paris F-94000, France
}

Correspondence should be addressed to Anne-Laure Bonnet; albonnet@hotmail.com

Received 24 January 2020; Revised 26 May 2020; Accepted 15 July 2020; Published 4 August 2020

Academic Editor: Balraj Mittal

Copyright (C) 2020 Anne-Laure Bonnet et al. This is an open access article distributed under the Creative Commons Attribution License, which permits unrestricted use, distribution, and reproduction in any medium, provided the original work is properly cited.

\begin{abstract}
Amelogenesis imperfecta (AI) represents rare tooth anomalies that affect the quality and/or quantity of the enamel. Clinical phenotypes display a wide spectrum, ranging from mild color changes to severe structural alterations with daily pain. However, all affect the quality of life because of mechanical, psychological, esthetic, and/or social repercussions. Several gene mutations have been linked to AI as a nonsyndromic (isolated) phenotype or a wider syndrome. This case report aimed to present a family with dental structure anomalies followed up in the dental department of the Louis Mourier Hospital (APHP, France) for their extremely poor dental condition. The proband and his mother were clinically diagnosed with AI, and genetic analysis revealed an already described variant in $D L X 3$. Then, the family was further examined for tricho-dento-osseous syndrome. This report illustrates the challenge of diagnosing dental structure anomalies, specifically AI, in adults and highlights the need for an accurate and accessible molecular diagnosis for those anomalies to discriminate between isolated and syndromic pathologies.
\end{abstract}

\section{Introduction}

Amelogenesis imperfecta (AI) is a rare dental disease affecting the enamel structure, with an estimated prevalence of $1 / 700$ to $1 / 14000$ (ORPHA disorder 88661), a large range due to a mis- or underevaluation of tooth structure anomalies and lack of large clinical studies on this topic. AI has been described for years following the Witkop classification [1], aiming to specify the different aspects, forms, and colors of enamel anomalies. The bedrock of this classification is the AI "macro" pathophysiology, namely, impairment of enamel secretion or maturation stages during amelogenesis. However, presently, it is recognized that (1) clinical evaluation of the dysmorphic enamel is biased as soon as a tooth erupts, due to attrition, erosion, and other intraoral phenomena and (2) phenotypes are heterogeneous and can overlap $[2,3]$.
Another crucial aspect when considering AI is that this anomaly can be inherited alone or with other anomalies within a wider syndrome, such as enamel renal syndrome associated with FAM20A mutations [4] and AI associated with vitamin D-resistant rickets linked to VDR or CYP27BA mutations [5]. Since 1998, DLX3 mutations have been associated with tricho-dento-osseous syndrome (TDO, OMIM\#190320) [6, 7]. TDO is described as a dominant inherited syndrome combining hypoplastic AI with taurodontism, head and neck skeletal anomalies (increased thickness, higher bone density, and obliteration of facial sinuses), and curly or kinky hair. Nail and skin anomalies are often associated with TDO [8-10]. Other dental defects, such as pulp obliteration, shorter and dysmorphic roots, and dental eruption disorders (early or delayed), have sometimes been reported [11]. Recent publications have highlighted the 
possibility of "attenuated" TDO [12-14], relaunching the debate on a common or distinct diagnosis between TDO and the hypoplastic-hypomature type with the taurodontism form of AI (OMIM \#104510), both linked to DLX3 mutations [15].

In direct line with this debate, here, we describe the unexpected diagnosis of the known DLX3 c.398G > C, p. Arg133 Pro, variant in a family followed for global dental rehabilitation by the dental department of Louis Mourier Hospital (Paris, France).

\section{Case Presentation}

A 10-year-old male African child presented at the emergency department of Louis Mourier Hospital with cellulitis related to necrosis of the maxillary first right permanent incisor. After 1 year, he returned for general consultation. He had no history of medical conditions, and no hair, skin, or nail defects had been noted by the clinicians or reported by the parents. Oral examination revealed a healthy mucosa without bone abnormalities. However, the teeth showed significant structural and eruption abnormalities. The enamel was dark yellow, streaked, and severely worn (Figure 1(a)). It appeared translucent in the areas of wear. The enamel radio-opacity was similar to that of dentin (Figure 1(b)). On orthopantomography (Figure 1(b)), taurodontism was observed on the first permanent molars. The tooth eruption was severely disturbed. The first permanent molars and mandibulary first left deciduous molar were impacted. Roots appeared shorter than normal. After several dental infections, dental extractions and complete denture removal were performed (Figure 1(c)).

The proband's mother had a reported diagnosis of "dentinogenesis imperfecta" associated with painless mandibular bone exostosis from a previous visit. Clinically, the enamel was orange-brown and appeared severely worn out (Figure 2(a)). Panoramic radiography confirmed multifocal areas of high density in the mandibular bone (Figure 2(b)). This clinical picture suggested osseous dysplasia. More specifically, the multifocal fibro-osseous dysplastic areas characterized by radio-opaque cementumlike masses allowed the clinician to make a diagnosis of florid cemento-osseous dysplasia [16]. Dental examination revealed teeth with thin or absent enamel, numerous endodontic treatments and restorations, and missing teeth (Figure 2(b)). Whole-body scintigraphy performed to further explore the bone status revealed only one point of hyperossification on the right ankle. Radiographic examination of the left hand performed after a single painful episode revealed only a faint modification of the fifth phalanx. Furthermore, there were no hair, skin, or nail defects, so no formal diagnosis of TDO was established [16]. Residual teeth (except for two mandibular molars, left and right) were extracted, and rehabilitation was performed using a complete maxillary and partial mandibulary removable denture. These oral conditions were not reported for the patient's brothers, aged 2 and 5 years (considering the limitation of the clinical examination regarding their ages), or father (Figure 3(a)).
A clinical diagnosis of AI, hypoplastic/hypocalcified, was established for the proband according to the criteria defined by Witkop [17]. Because of the proband's teeth features, along with the mother's contradictory former diagnosis of dentinogenesis imperfecta, genetic exploration was indicated.

2.1. Genetic Material and Methods. DNA from patients I.1 and II.1 were analyzed at the Department of Molecular Biology and Genetics of Cochin Hospital (APHP, Paris) (Figure 3(a)). High-throughput targeted sequencing was performed on an ion $\mathrm{PGM}^{\mathrm{TM}}$ system from amplicon libraries (Thermo Fisher Scientific) (Table 1). Bioinformatics analysis relied on Thermo Fisher Scientific tools and the homemade pipeline Polydiag of the Paris Descartes University, Imagine Institute bioinformatics platform. A pathogenic variant in $D L X 3$, exon 2, c.398G > C, p. Arg133Pro, was identified in both patients I.1 and II.1, but not in I.2 (DNAs for II.2 and II.3 were not available) (Figure 3(b)), neither in a positive control cohort of patients with AI nor in the database of the Department of Molecular Biology and Genetics [15].

The substitution c.398G>C was reported by Nieminen et al. in 2011 but, then, associated with a typical TDO family. It encodes for a missense at the protein level, replacing the arginine in position 133 with a proline at the beginning of the homeodomain of the DLX3 protein. Predictors related to consensus sequence conservation interspecies (Polyphen2) and 3D structure/missense disruption (SIFT, Mutation Taster) annotate this substitution as deleterious or pathogenic. Hence, this variant was considered a class $\mathrm{V}$ variant (pathogenic), following the American College of Medical Genetics and Genomics recommendations [18]. Considering all these clinical, radiographic, and molecular elements, a diagnosis of "attenuated" TDO was proposed for the proband and his mother.

\section{Discussion}

AI is a highly variable tooth disorder that involves the enamel structure. Its management requires a multidisciplinary team; specialists in medical genetics, mainly in rare dental diseases; and restorative and prosthodontics experts. Presently, the diagnosis of AI still appears difficult for dentists. Clinical descriptions and the classification established by Witkop are complicated and not well applicable because of the great variability of the AI phenotype [17].

Classically, clinicians use hypocalcified, hypomature, or hypoplastic terms to describe AI. Hypoplastic forms are used when the enamel shows reduced thickness. The tooth surfaces can be smooth or rough. The hypoplastic form is mainly associated with an anomaly of enamel matrix secretion [3]. A hypomature enamel is described as opaque, white to yellow-brown, hard, and easily detachable from the dentin and has a normal thickness. These signs are linked to a lack of protein elimination in the extracellular matrix during the enamel maturation phase. Less mechanical strength is observed. The hypocalcified form is characterized 


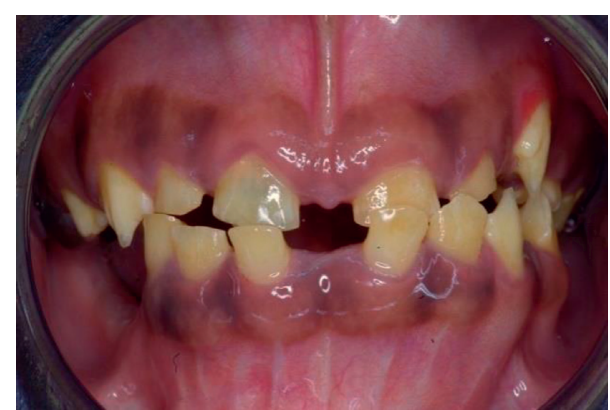

(a)

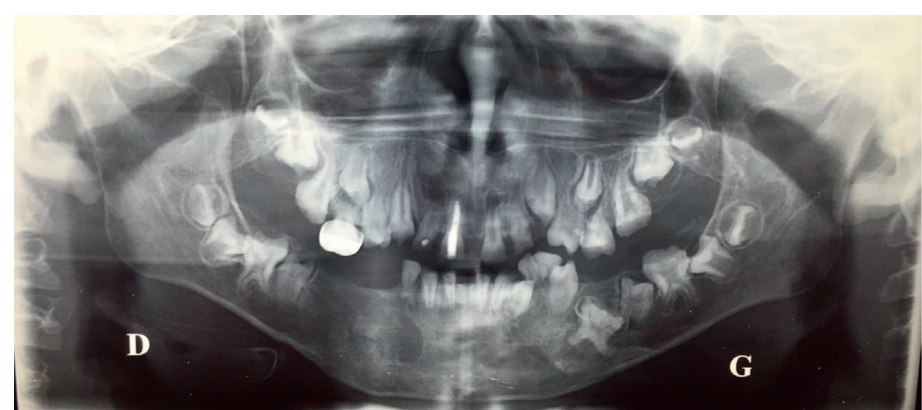

(b)

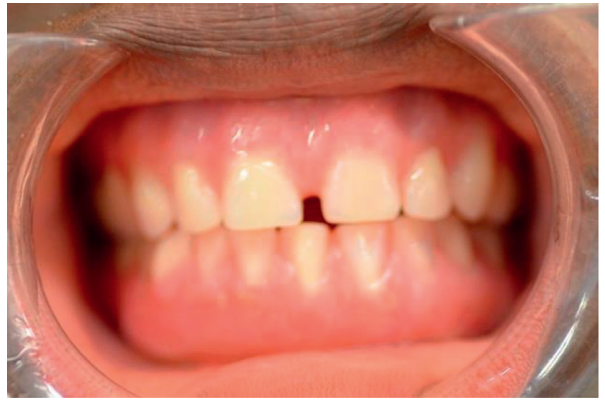

(c)

FIGURE 1: Clinical and radiological views of the proband. (a) Intraoral photograph of the patient showing hypocalcified enamel and eruption anomalies. The enamel is dark yellow, streaked, and severely worn out. The proband's teeth are in the transitional dentition phase, with major diastemas. (b) Panoramic radiograph of the proband showing retained, impacted, and missing teeth; generally thin or absent enamel; taurodontism on first permanent molars; one temporary molar restored with a preformed metallic crown; and the central right incisor endodontically treated. (c) Intraoral photograph immediately after prosthetic rehabilitation of the patient with a complete removable denture. An anterior disastema is preserved for esthetics.

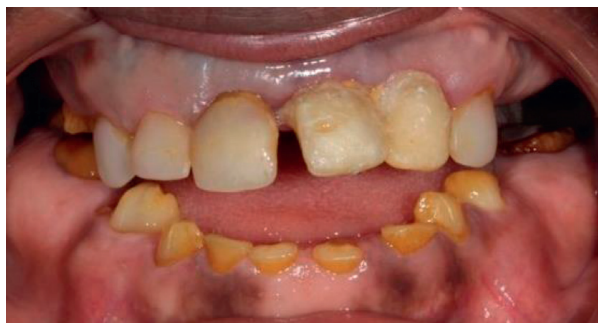

(a)

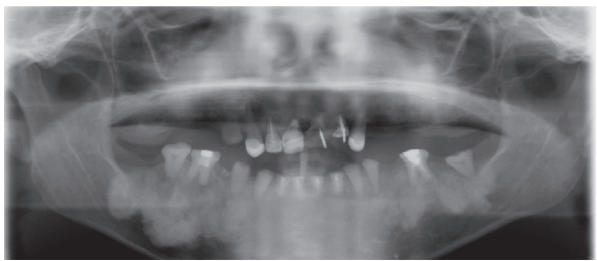

(b)

Figure 2: (a) Clinical view of the proband's mother during the first examination. On the upper jaw, all teeth are covered by prosthetic elements. On the lower jaw, the remaining teeth present severe wear with $>50 \%$ loss of tooth structure. The enamel is orange-brown. (b) Panoramic radiograph of the proband's mother showing mandibular high bone density, missing teeth, absent enamel, and many restored and endodontically treated teeth.

by impaired mineralization of enamel crystallites during the secretion phase. It results in a creamy-white to yellow-brown rough enamel surface. The enamel is generally normal in thickness on newly erupted teeth but rapidly tends to be chipped away or scraped from the dentin. Radiographically, enamel radio-opacity appears similar to that of the dentin [17].

Over the years, all associations of clinical AI forms have been reported. Recent studies have highlighted the cellular and molecular levels of pathophysiology knowledge, and the common and global term of AI is proposed for all clinical forms $[19,20]$. The modern approach to AI tends to regroup causative proteins depending on their function and pathway. Different protein groups can be mentioned, even though all the AI causative genes have not yet been discovered. The first described were the enamel matrix proteins (AMELX, ENAM), enamel matrix proteases (MMP20, KLK4), and proteins involved in cell-cell and cell-matrix adhesion (AMTN, COL17A1, LAMA3, LAMB3, ITGB6, and FAM83H), transport (WDR72, SLC24A4), and control (even if not yet well defined) of amelogenesis (GPR68, ODAPH, ACPT, FAM20A, and DLX3) [3]. 


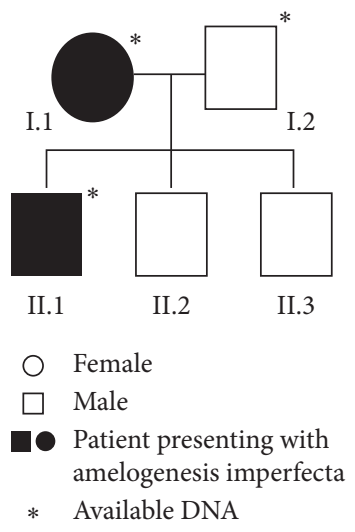

(a)

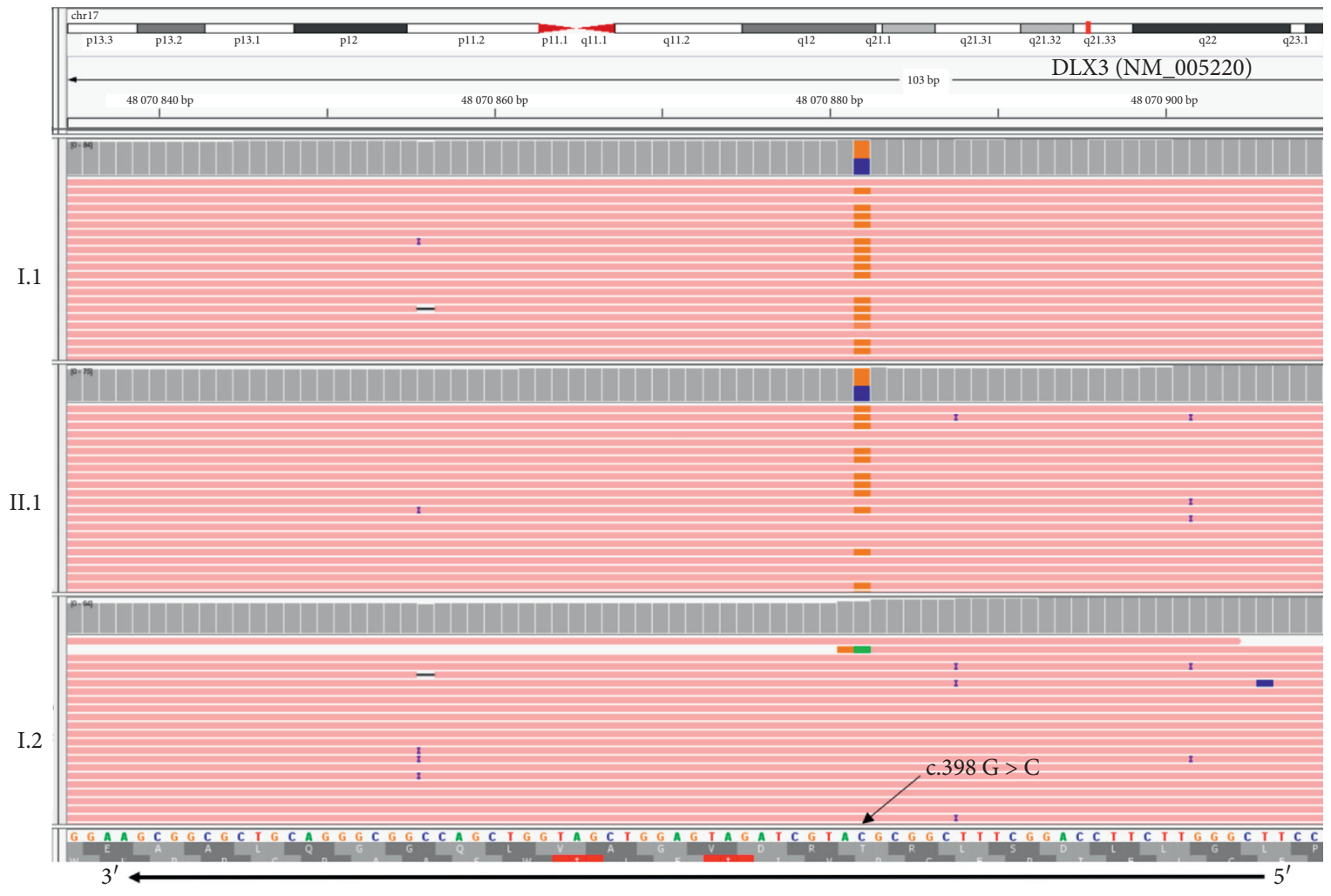

(b)

Figure 3: (a) Family pedigree. (b) DLX3 NM_005220 alignment on the Integrative Genomics Viewer (IGV, Broad Institute), reference genome hg19, after next-generation sequencing on a targeted panel for amelogenesis imperfecta. Upper tracks, patient I.1; middle tracks, patient II.1; and bottom tracks, patient I.2. Visualization of misalignments in orange versus pink are due to the heterozygous frameshift $\mathrm{G}>\mathrm{C}$ at position 398 (direct lecture $\mathrm{C}>\mathrm{G}$ on reverse strand).

Therefore, this new AI approach is no longer only clinical but also biological. This requires a strong network between geneticists and specialists of rare dental diseases to improve the correlation between genotype and phenotype and close collaboration with restorative treatment experts. This network could enhance treatment options, notably an individual therapeutic approach, such as an adaptive adhesive technique, in case of MMP20 mutation [21] or new protocols to systematically and preventively recover all cuspid teeth with $L A M A 3$ or FAM83H mutation because of the rapid and widespread loss of enamel associated with malfunction of these proteins.
Another important aspect is to consider the isolated character or not of AI. In the described family, dental problems, even though the first proposed diagnosis based on the clinical observation of the proband's mother was inaccurate, were very well known by all family members. The proband had been supported and accompanied by his parents since his early childhood, and dental care, even if not fully appropriate, had been provided. However, a complete and definite diagnosis without a molecular approach remains difficult. When dental structure anomalies are observed for the first time in adulthood, diagnosis is almost 
TABLE 1: Targeted genes panel for enamel structure anomalies.

\begin{tabular}{|c|c|c|c|}
\hline Protein & Gene (HGNC) & Localization & ENST \\
\hline Amelogenin & AMELX & $\mathrm{Xp} 22.31-22.1$ & ENST0000380712.7 \\
\hline Enamelin & ENAM & $4 q 13.3$ & ENST00000396073.3 \\
\hline Ameloblastin & $\mathrm{AMBN}$ & $4 \mathrm{q} 21$ & ENST00000922937.10 \\
\hline Distal less homeobox 3 & DLX3 & $17 \mathrm{q} 21$ & ENST00000434704.2 \\
\hline Integrin, beta 6 & ITGB6 & $2 \mathrm{q} 24.2$ & ENST00000283249.6 \\
\hline laminin, beta 3 & LAMB3 & $1 \mathrm{q} 32$ & ENST00000356082.8 \\
\hline Amelotin & AMTN & $4 \mathrm{q} 13.3$ & ENST00000339336.8 \\
\hline WD repeat domain 72 & WDR72 & $15 \mathrm{q} 21.3$ & ENST00000360509.9 \\
\hline Matrix metallopeptidase 20 & MMP20 & $11 \mathrm{q} 22.3$ & ENST00000260228.2 \\
\hline Solute carrier family $24 \mathrm{~A} 4$ & SLC24A4 & $14 \mathrm{q} 32.12$ & ENST00000532405.5 \\
\hline Kallikrein-related peptidase 4 & KLK4 & $19 \mathrm{q} 13.41$ & ENST00000324041.5 \\
\hline Bone morphogenetic phosphoprotein 2 & BMP2 & $20 \mathrm{p} 13$ & ENST00000378827.4 \\
\hline Odontogenesis-associated phosphoprotein & ODAPH & $4 q 21.1$ & ENST00000311623.8 \\
\hline Collagen 17 alpha 1 & COL17A1 & $10 \mathrm{q} 24.3$ & ENST00000353479.9 \\
\hline Cyclin and CBS domain divalent metal cation transport mediator 4 & CNNM4 & $2 \mathrm{q} 11.2$ & ENST00000377075.2 \\
\hline Family with sequence similarity 83 , member $\mathrm{H}$ & FAM83H & $8 \mathrm{q} 24.3$ & ENST00000388913.3 \\
\hline Laminin subunit alpha 3 & LAMA3 & $18 \mathrm{q} 11$ & ENST00000313654.13 \\
\hline Acid phosphatase 4 & ACP4 & $19 q 13.33$ & ENST00000270593.1 \\
\hline G-protein-coupled receptor 68 & GPR68 & $14 \mathrm{q} 32$ & ENST00000531499.2 \\
\hline
\end{tabular}

impossible because of posteruptive modifications and sequelae. The second aspect agrees with recent studies on AI topics, highlighting that the frontier between syndromic and nonsyndromic forms of AI is not so watertight. In this report, except for the osseous mandibular densities in the proband's mother (which, when observed in middle-aged African women, could be diagnosed as florid cemento-osseous dysplasia), only remarkably faint signs usually linked to TDO had been noted by the family or pediatricians or were even noticeable by dental surgeon specialists of rare diseases. When the $D L X 3$ mutation diagnosis was made, the whole family had received a complete explanation on TDO and had been advised to undergo extensive testing for DLX3 mutation holders and their offspring.

Specifically, the clinical diagnosis of patient II.2 should be reassessed over time. Indeed, when comparing our family with the Finnish family first described as carrying the c.398G > C frameshift [11], the clinical features of the Finnish patients were stronger for a TDO diagnosis (association of dental, facial bone, and hair phenotypes). However, one cannot ignore the mandibular ossification in patient I.1 and the severely disturbed eruption associated with shortened roots and taurodontism in patient II.2 that resembled those of Finnish patients and supported an "attenuated" TDO diagnosis.

\section{Conclusions}

Our report illustrates the challenge of diagnosing dental structure anomalies and, specifically, AI in adults and highlights the need for an accurate and accessible molecular diagnosis for these anomalies to (1) ameliorate the patient's course and provide appropriate care and (2) provide a better understanding of the underlying pathophysiology. It is essential to obtain this knowledge to assure the patient that he or she will not pass on a syndromic trait to the offspring. In France, this process also helps patients receive financial support for dental or medical care. Alongside the molecular aspect, self-esteem, wellness, and social aspects of the pathology should be better considered.

\section{Consent}

Written informed consent was obtained from the patient's parents for publication of this case report, and any potentially identifying information was removed. Gene sequencing was performed after written informed consent was provided by the patient's parents for themselves and their child.

\section{Disclosure}

Anne-Margaux Collignon and Celine Gaucher are co-last authors.

\section{Conflicts of Interest}

The authors declare that there are no conflicts of interest regarding the publication of this article.

\section{Authors' Contributions}

$\mathrm{KS}, \mathrm{AMC}$, and AW were in charge of the clinical follow-up of the patient. ALB, AMC, and CG drafted the manuscript. CS and $C G$ performed gene sequencing and molecular analysis. AMC and CG reviewed the manuscript and made substantial contributions to data interpretation. All authors approved the final version of the manuscript. Anne-Margaux Collignon and Céline Gaucher contributed equally to the work.

\section{Acknowledgments}

The authors would like to thank Patrick Nitschké and Audrey Briand-Suleau for the bioinformatic pipeline 
Polydiag of Paris Descartes University, Imagine Institute bioinformatics platform, Chrystel Leroy for the technical support, Pr Michel Vidaud, head of the Genetic and Molecular Biology Department of Cochin Hospital, APHP Centre, Paris, Claudine Wulfman and Michel Postaire for the clinical view and prosthodontic care of the proband's mother, Thong Nguyen and Sibylle Vital for addressing the patient for molecular analysis, and Pr Marysette Folliguet, Chief of the Department of Odontology, AP-HP, Nord, Université de Paris, Hospital Louis Mourier.

\section{References}

[1] C. J. Witkop, "Clinical aspects of dental anomalies," International Dental Journal, vol. 26, no. 4, pp. 378-390, 1976.

[2] J. T. Wright, M. Torain, K. Long et al., "Amelogenesis imperfecta: genotype-phenotype studies in 71 families," Cells Tissues Organs, vol. 194, no. 2-4, pp. 279-283, 2011.

[3] C. E. L. Smith, J. A. Poulter, A. Antanaviciute et al., "Amelogenesis imperfecta; genes, proteins, and pathways," Frontiers in Physiology, vol. 8, p. 435, 2017.

[4] G. Jaureguiberry, M. De la Dure-Molla, D. Parry et al., "Nephrocalcinosis (enamel renal syndrome) caused by autosomal RecessiveFAM20AMutations," Nephron Physiology, vol. 122, no. 1-2, pp. 1-6, 2012.

[5] A. E. Hanna, S. Sanjad, R. Andary, G. Nemer, and J. G. Ghafari, "Tooth development associated with mutations in hereditary vitamin D-resistant rickets," JDR Clinical \& Translational Research, vol. 3, no. 1, pp. 28-34, 2018.

[6] J. Price, D. W. Bowden, J. T. Wright, M. J. Pettenati, and T. C. Hart, "Identification of a mutation in DLX3 associated with tricho-dento- osseous (TDO) syndrome," Human Molecular Genetics, vol. 7, no. 3, pp. 563-569, 1998.

[7] J. A. Price, J. T. Wright, K. Kula, D. W. Bowden, and T. C. Hart, "A common DLX3 gene mutation is responsible for tricho-dento-osseous syndrome in Virginia and North Carolina families," Journal of Medical Genetics, vol. 35, no. 10, pp. 825-828, 1998.

[8] D. E. Y. Mayer, C. Baal, M. Litschauer-Poursadrollah, W. Hemmer, and R. Jarisch, "Unkämmbare Haare und atopische Dermatitis bei tricho-dentoossärem Syndrom," JDDG: Journal der Deutschen Dermatologischen Gesellschaft, vol. 8, no. 2, pp. 102-104, 2010.

[9] F. Quattromani, S. D. Shapiro, R. S. Young et al., "Clinical heterogeneity in the tricho-dento-osseous syndrome," $\mathrm{Hu}$ man Genetics, vol. 64, no. 2, pp. 116-121, 1983.

[10] J. T. Wright, K. Kula, K. Hall, J. H. Simmons, and T. C. Hart, "Analysis of the tricho-dento-osseous syndrome genotype and phenotype," American Journal of Medical Genetics, vol. 72, no. 2, pp. 197-204, 1997.

[11] P. Nieminen, P.-L. Lukinmaa, H. Alapulli et al., "DLX3 homeodomain mutations cause tricho-dento-osseous syndrome with novel phenotypes," Cells Tissues Organs, vol. 194, no. 1, pp. 49-59, 2011.

[12] J. T. Wright, S. P. Hong, D. Simmons et al., "DLX3 c.561_ 562delCT mutation causes attenuated phenotype of trichodento-osseous syndrome," American Journal of Medical Genetics Part A, vol. 146, no. 3, pp. 343-349, 2008.

[13] Y.-J. Kim, F. Seymen, M. Koruyucu et al., "Unexpected identification of a recurrent mutation in theDLX3gene causing amelogenesis imperfecta," Oral Diseases, vol. 22, no. 4, pp. 297-302, 2016.
[14] L. L. E. Whitehouse, C. E. L. Smith, J. A. Poulter et al., "Novel DLX3 variants in amelogenesis imperfecta with attenuated tricho-dento-osseous syndrome," Oral Diseases, vol. 25, no. 1, pp. 182-191, 2019.

[15] J. Price, J. Wright, S. Walker, P. Crawford, M. Aldred, and T. Hart, "Tricho-dento-osseous syndrome and amelogenesis imperfecta with taurodontism are genetically distinct conditions," Clinical Genetics, vol. 56, no. 1, pp. 35-40, 1999.

[16] S. Fenerty, W. Shaw, R. Verma et al., "Florid cemento-osseous dysplasia: review of an uncommon fibro-osseous lesion of the jaw with important clinical implications," Skeletal Radiology, vol. 46, no. 5, pp. 581-590, 2017.

[17] C. J. Witkop, "Amelogenesis imperfecta, dentinogenesis imperfecta and dentin dysplasia revisited: problems in classification," Journal of Oral Pathology and Medicine, vol. 17, no. 9-10, pp. 547-553, 1988.

[18] S. Richards, N. Aziz, N. Aziz et al., "Standards and guidelines for the interpretation of sequence variants: a joint consensus recommendation of the American College of medical genetics and Genomics and the association for molecular pathology," Genetics in Medicine, vol. 17, no. 5, pp. 405-423, 2015.

[19] H.-C. Chan, N. M. R. P. Estrella, R. N. Milkovich, J.-W. Kim, J. P. Simmer, and J. C.-C. Hu, "Target gene analyses of 39 amelogenesis imperfecta kindreds," European Journal of Oral Sciences, vol. 119, no. 1, pp. 311-323, 2011.

[20] J. T. Wright, I. A. Carrion, and C. Morris, "The molecular basis of hereditary enamel defects in humans," Journal of Dental Research, vol. 94, no. 1, pp. 52-61, 2015.

[21] D. J. Epasinghe and C. K. Y. Yiu, "Effect of etching on bonding of a self-etch adhesive to dentine affected by amelogenesis imperfecta," Journal of Investigative and Clinical Dentistry, vol. 9, no. 1, 2018. 